\title{
USO Y PRESENCIA DE LAS TECNOLOGÍA EN LAS PERSONAS CON DISCAPACIDAD INTELECTUAL Y DEL DESARROLLO. HERRAMIENTAS DIGITALES EN TIEMPOS DE CRISIS
}

\author{
Brizeida Hernández Sánchez \\ Universidad Especializada de las Américas \\ brizeida.hernandez@udelas.ac.pa \\ José Carlos Sánchez García \\ Universidad de Salamanca \\ jsanchez@usal.es \\ Greisy González Cedeño \\ Universidad Especializada de las Américas. Panamá \\ greisy.gonzalez.1992@udelas.ac.pa
}

Recepción Artículo: 04 junio 2021

Admisión Evaluación: 04 junio 2021

Informe Evaluador 1: 04 junio 2021

Informe Evaluador 2: 04 junio 2021

Aprobación Publicación: 04 junio 2021

\section{RESUMEN}

El objetivo de esta investigación identificó la presencia y utilidad de las herramientas digitales en las personas con discapacidad y del desarrollo (DID), se buscó conocer qué herramientas les darían ventajas, qué herramientas necesitan para trabajar; además, se identificó las necesidades de formación. Estos objetivos nos llevaron a planearnos supuestos: En qué medida competencias digitales inciden en las personas con discapacidad y del desarrollo hacia la inserción laboral. Si estas inciden en su inserción laboral. La metodología de la investigación es cuantitativa con un diseño cuasiexperimento, han participado 257 personas con discapacidad, nueve provincias. 51,7\% masculinos, 48 . 2\% femenino. Se elaboró un instrumento tipo encuesta dirigido a personas con discapacidad. La elaboración del instrumento se realizó a partir de las siguientes fuentes: a) revisión de la literatura científica sobre discapacidad y tecnología y su impacto en la inserción laboral y los programas; b) informe de las asociaciones que trabajan para, con y por las personas con discapacidad intelectual y c) repositorios que recopilan información sobre actividades de las personas con discapacidad. La técnica fue por Ilamadas de teléfono usando la aplicación WatsApp aplicada entre el 18 de diciembre 2020 a 28 febrero 2021. Entre los aspectos evaluados están: tecnología de uso diario; dispositivos y usabilidad o retos de la tecnología para las personas con discapacidad. Los primeros resultados expresan que $57,5 \%$ han tenido alguna experiencia laboral y $42,4 \%$ no tiene ninguna relación con el empleo. Las situaciones recogidas nos permiten diseñar un mapa completo en relación, si las tecnologías ayudan e impactan en la calidad de vida.

Palabras claves: tecnologías; discapacidad; equidad; asociaciones; calidad de vida 


\begin{abstract}
Use and presence of technology in people with intellectual and developmental disabilities. digital tools in times of crisis. The objective of this research identified the presence and usefulness of digital tools in people with disabilities and development (DID), it sought to know what tools would give them advantages, what tools they need to work; in addition, training needs were identified. These objectives led us to plan assumptions: To what extent digital skills affect people with disabilities and their development towards employment. If these affect your job placement. The research methodology is quantitative with a quasi-experiment design, 257 people with disabilities have participated, from nine provinces. $51.7 \%$ male, $48.2 \%$ female. A survey-type instrument was developed for people with disabilities. The development of the instrument was carried out from the following sources: a) review of the scientific literature on disability and technology and its impact on job placement and programs; b) report of associations that work for, with and for people with intellectual disabilities and c) repositories that collect information on activities of people with disabilities. The technique was by phone calls using the WatsApp application applied between December 18, 2020, to February 28, 2021. The aspects evaluated are technology for daily use; devices and usability or challenges of technology for people with disabilities. The first results express that $57.5 \%$ have had some work experience and $42.4 \%$ have no relationship with employment. The collected situations allow us to design a complete map in relation to whether the technologies help and impact the quality of life.
\end{abstract}

Keywords: technologies; disability; equity; associations; quality of life

\title{
INTRODUCCIÓN
}

Las tecnologías de la información y la comunicación (TIC) han permeado los sistemas escolares de todo el mundo con la promesa de mejorarlos (Sunkel, 2012). En una sociedad en constante cambio, se destaca el desarrollo tecnológico y la educación debe actualizarse y responder a los desafíos de este desarrollo. Analizando el papel de las TIC en el aprendizaje de los estudiantes en general, es pertinente plantearse la siguiente pregunta: ¿cómo se pueden utilizar las TIC para apoyar una filosofía pedagógica inclusiva, con miras a una 'escuela para todos'? Varios autores (ej: Santos, 2006) reconocen perspectivas positivas sobre el uso de las TIC, en relación con personas con cierta discapacidad, a nivel físico o mental, a saber: contribuir a una normalización de su vida, desarrollo de capacidades intelectuales, facilitar el acceso al conocimiento y al aprendizaje, al ocio y a evitar la exclusión, que en el futuro también les permitirá integrarse en el contexto laboral.

Al transitar al ámbito laboral, automáticamente regresamos a la dimensión educativa, ya que este es uno de los primeros pasos hacia la construcción del ser social, donde se desarrollan valores, competencias y habilidades para el ejercicio de una actividad laboral (Siles- Rojas et al.2020). Así, la inclusión en el mercado laboral es un proceso complejo para todos, especialmente para las personas con discapacidad, debido a los prejuicios que crean numerosas barreras. El empleo inclusivo es la actividad laboral dirigida y adaptada a personas que padecen algún tipo de discapacidad, intelectual, física 0 emocional.

Es necesario aclarar los conceptos de "empleo inclusivo "y "empresa inclusiva. "El primero se refiere a la implementación de medidas multidisciplinares enfocadas a mejorar la empleabilidad de un grupo de personas con peculiaridades, que les impiden competir en el mercado laboral con las mismas ventajas que el resto. El segundo concepto se utiliza para referirse a los negocios que implican un grupo con dificultades financieras, pero que no necesariamente sufren alguna limitación.

Los avances tecnológicos han permitido abrir tres caminos imprescindibles para la inclusión: 1) la oferta de cursos presenciales o formaciones a distancia dirigidas a mejorar la cualificación de este colectivo en la gestión o gestión empresarial; 2) la introducción y popularización del teletrabajo, algo que ha ganado protagonismo durante la pandemia; y 3) la búsqueda de empleo específicamente dirigido a personas con discapacidad. Y es precisamente en este tercer punto donde se centran las propuestas para promover el empleo inclusivo.

La inclusión de las personas con discapacidad en el mercado laboral se entiende como un factor decisivo para la inclusión social, la independencia económica y la consecuente valorización y realización de estos ciuda- 
danos. Las personas con discapacidad se mencionan en varias partes de los Objetivos de Desarrollo Sostenible y, específicamente, en las partes relacionadas con la educación, el crecimiento y el empleo, la desigualdad, la accesibilidad de los asentamientos humanos. De los 17 objetivos de la Agenda 2030, podemos destacar los siguientes relacionados con la discapacidad: Objetivo 4: Educación inclusiva y equitativa de calidad y promoción de oportunidades de aprendizaje a lo largo de la vida para todos. Además, la propuesta pide la construcción y mejora de instalaciones educativas que sean sensibles a los niños, las discapacidades y el género ; Objetivo 8 : promover el crecimiento económico sostenido, inclusivo y sostenible, el empleo pleno y productivo y el trabajo decente para todos, incluidas las personas con discapacidad; Objetivo 10 , que se esfuerza por reducir la desigualdad dentro y entre los países mediante el empoderamiento y la promoción de la inclusión social, económica y política de todos, incluidas las personas con discapacidad ; Objetivo 11 : proporcionar acceso a sistemas de transporte seguros, asequibles, accesibles y sostenibles, mejorando la seguridad vial, en particular mediante la expansión del transporte público, con especial atención a las necesidades de las personas en situaciones vulnerables, como las personas con discapacidad; y el Objetivo 17: aumento de la calidad y la puntualidad de los datos desglosados por discapacidad.

La velocidad de desarrollo de la sociedad de la información actual se ve como una desventaja para las personas con discapacidad, ya que requiere una mayor eficiencia en el tratamiento e interpretación de la información. Las tecnologías de la información y la comunicación (TIC) son hoy en día la ruta que seguir para la transformación social y económica. El uso de la tecnología en personas con discapacidad puede considerarse otro aspecto del ajuste a sus necesidades (Tanis et al., 2012). En los últimos años, las TIC se han utilizado como una herramienta para mejorar la calidad de vida de las personas de una forma más eficiente y eficaz. Existen varias herramientas que ayudan a las personas con discapacidad, brindándoles oportunidades de aprendizaje, aumentando su potencial en diferentes ámbitos de sus vidas, al difuminar las desigualdades (Mishra et al., 2010). Estas tecnologías dan mayor libertad a las personas con discapacidad y a sus familias, ya que las personas con discapacidad pueden ser más independientes, aumentando su calidad de vida (Lorah et al., 2015).

Las TIC son una gran esperanza para las personas con discapacidad: pueden reducir las barreras que pueden perjudicar 0 impedir que las personas con discapacidad participen en las actividades cotidianas (D'Aubin, 2007). Las tecnologías han adquirido un significado especial en la mejora y normalización de la vida de las personas con discapacidad, así como en su acceso al empleo, permitiéndoles desarrollarse, tanto a nivel personal como laboral. Desde este punto de vista, las tecnologías de la información y la comunicación se han convertido en importantes aliados para promover su plena inclusión (Zúñiga et al., 2016). Por ejemplo, existen aplicaciones especialmente diseñadas para personas con discapacidad que, además de facilitar acciones en su día a día, como buscar trabajo a través de Internet, las conectan a un entorno cada vez más digital.

Las TIC abren muchas posibilidades, aunque pueden generar un nuevo tipo de exclusión porque parte de la población puede acceder a estas tecnologías de forma más temprana y eficiente, provocando distinciones entre las personas que pueden aprovechar fácilmente estos avances y las personas que tienen un acceso limitado por carencia. de medios o conocimientos sobre cómo utilizarlos (Berrío Zapata et al., 2020).

Los cambios en la sociedad provocados por las TIC, internet y las redes sociales han modificado los estilos de vida, la organización y la convivencia (Balladares -Burgos, 2019). La inclusión digital se puede definir como un derecho de tercera generación que proviene de los cambios tecnológicos, el avance de la ciencia y su impacto en la vida humana (Chacón et al., 2017) que otros consideran derechos de cuarta generación en el momento del surgimiento del código abierto para la educación. y desarrollo comunitario (UNESCO, 2009). La inclusión digital como derecho humano busca la promoción de la justicia social y, para ello, requiere de la colaboración democrática de todos, la generación de procesos innovadores, un diálogo intercultural constructivo y la participación internacional (López y Samek, 2009), como estaba previsto en el 2030 Agenda.

Barlott y col. (2019) señalan que la interacción entre oportunidad social y habilidades personales potencia la inclusión digital de personas con discapacidad. Por lo tanto, las TIC abren puertas a nuevas oportunidades y 
prometen empoderar a las personas con discapacidad en la misma medida que a sus pares sin discapacidad (Anderberg y Jönsson, 2005).

Las personas con discapacidad no son un grupo homogéneo, es decir, lo que incluye a un grupo excluye al otro. Por ejemplo, las personas con discapacidad intelectual tienen una desventaja particular cuando se trata de planificar o trabajar en equipo. Los cambios en la sociedad también han modificado el significado de accesibilidad, no solo refiriéndose a la eliminación de barreras físicas, sino también a la adaptación y flexibilidad del hardware y software de las TIC para llegar a cualquier usuario, sean cuales sean sus limitaciones (Benavides et al., 2018).

Las TIC brindan a las personas con discapacidad los apoyos necesarios para tener una educación a la altura de las personas sin discapacidad y para poder participar activamente en su sociedad (Alper y Goggin, 2017). Esto otorga a las personas con discapacidad una mayor autonomía económica e inclusión social (Khanlou et al., 2021).

Las personas con discapacidad '(...) incluyen aquellas que enfrentan retos físicos, intelectuales o sensoriales a largo plazo que en interacción con diversas barreras pueden obstaculizar su participación plena y efectiva en la sociedad en igualdad de condiciones con los demás' (Leonardi et al. 2006, p. 1220), según la definición de las Naciones Unidas (ONU). Las personas con discapacidad no solo tienen que hacer frente a las barreras producidas por un mercado laboral competitivo e inestable, también deben superar las barreras adicionales que son el resultado de sus circunstancias personales (Michailakis, 2001). Por otro lado, el avance del desarrollo de las TIC ha abierto muchas oportunidades para las personas con discapacidad, también en lo que respecta al mercado laboral. Las TIC proporcionan un modelo para permitir que una persona con discapacidad se integre mejor social y económicamente en su comunidad (Khetarpal, 2016). El concepto de inclusión laboral designa la supresión de todas las barreras físicas y actitudes discriminatorias, permitiendo que las personas con discapacidad tengan acceso al mundo laboral y, en consecuencia, oportunidades de desarrollo personal, social, educativo y profesional (Leão y Silva, 2012).

Según datos de la Organización Internacional del Trabajo (OIT), en 2011, 60 millones de ciudadanos con discapacidad y / 0 discapacidad, en edad de trabajar, están desempleados . En 2017 Panamá tenía más de 400.000 personas con discapacidad. Hasta la fecha, más de 3.000 personas con discapacidad estaban empleadas, particularmente en la administración pública, donde trabajaban más de 700 personas con discapacidad (Naciones Unidas, 2017). En 2019, se promulgó el Decreto Ejecutivo $N^{\circ} 333$ de 5 de diciembre para reglamentar la Ley N - 15 de 31 de mayo de 2016, que brinda igualdad de oportunidades a las personas con discapacidad en la República de Panamá. Este reglamento tiene como objetivo, entre otros, la accesibilidad a la educación, la vivienda, la salud, el transporte, la cultura. Este Decreto también otorga a este grupo de empleados el doble del tiempo anual de baja por enfermedad.

Las TIC forman parte integrante del ámbito privado y profesional de personas de todos los rincones del mundo y están promoviendo un nuevo paradigma de sociedad, que puede facilitar la integración de las personas con discapacidad en la vida laboral. En cuanto al trabajo y empleo de las personas con discapacidad, además de lograr la igualdad a través de la accesibilidad, la Convención sobre los Derechos de las Personas con Discapacidad (2006) refuerza en su artículo 27, la necesidad de que el sector público emplee a personas con discapacidad y promueva el empleo. en el sector privado y, por lo tanto, pueden incluir sus propias políticas y medidas. Así, la CDPD (2006) especifica que este derecho concierne a la posibilidad de que las personas con discapacidad mantengan un trabajo de su libre elección y aceptado en el mundo laboral, en un entorno inclusivo y accesible.

Finalmente, es importante que exista una formación adecuada en el uso de las TIC y, en muchos casos, se requiere una formación a domicilio. Sin embargo, el teletrabajo no es un escenario perfecto, ya que las personas con discapacidad estarán socialmente aisladas. Estar empleado es una condición necesaria y crucial para el reconocimiento y la aceptación social, para la autonomía personal y económica y, particularmente en este caso, para la inclusión. 


\section{OBJETIVOS DE LA INVESTIGACIÓN}

El estudio identificó la presencia y utilidad de las herramientas digitales en las personas con discapacidad, se buscó conocer qué herramientas les darían ventajas, qué herramientas necesitan para trabajar; además, se identificó las necesidades de formación. Estos objetivos nos llevaron a planearnos supuestos: En qué medida competencias digitales inciden en las personas con discapacidad. Si estas inciden en su inserción laboral. El momento es crucial para responder a una población invisible que requiere de forma impostergable oportunidades para emplearse, desde un plan de vida y aporte a su comunidad. En marzo de 2020 se declara el Estado de Emergencia por causa de la pandemia por COVID-19 y se lanzan normativas de comunicación a favor de las personas con discapacidad (McKinney, y Swartz,2020) y se revelan grandes desigualdades en el acceso a la comunicación, discriminación, la falta de empleo, ausencia a la educación por ausencia de recursos.

\section{MUESTRA Y/O PARTICIPANTES}

La metodología de la investigación es cuantitativa con un diseño cuasiexperimento, han participado personas con discapacidad, nueve provincias. 51,7\% masculinos, 48. 2\% femenino. Se elaboró un instrumento tipo encuesta dirigido a personas con discapacidad. La elaboración del instrumento se realizó a partir de las siguientes fuentes: a) revisión de la literatura cientíica sobre discapacidad y tecnología y su impacto en la inserción laboral y los programas; y b) informe de las asociaciones que trabajan para, con y por las personas con discapacidad intelectual.

\section{METODOLOGÍA Y/O INSTRUMENTOS UTILIZADOS}

La técnica de recolección fue por llamadas de teléfono usando la aplicación WhatsApp aplicada entre el 18 de diciembre 2020 a 28 febrero 2021. El objetivo principal del estudio fue valorar cómo indicen las herramientas digitales en la persona con discapacidad. Por esta razón, el instrumento se enfocó en recoger datos en las siguientes categorías

- Preguntas generales

- Tecnología de uso diario.

- Dispositivos y usabilidad.

- Retos de la tecnología para las personas con discapacidad.

- Obstáculos de la tecnología para las personas con discapacidad.

- Herramientas digitales para el trabajo

- Programas de formación existentes

El cuestionario tiene un lenguaje pertinente y apropiado que permita la comprensión de los participantes. La población de estudio estará conformada por personas con discapacidad de la Federación Nacional Amigos de personas con discapacidad de la República de Panamá. Para la selección de la muestra se realizó un muestreo de tipo probabilístico. Este tipo de estudio mantiene, semejanzas con el muestreo aleatorio estratificado, pero no mantiene el carácter de aleatoriedad. Se extraerá una muestra también base a las variables de, sexo, edad, contexto, experiencias previas.

El procedimiento de diseño y contenido del instrumento fue validado por el Comité Ético del Instituto Nacional de Medicina Física y de Rehabilitación (INMFR). De conformidad con los principios de la Declaración de Helsinki. La encuesta elaborada por el equipo de investigación con el apoyo de Inclusión Panamá, fueron notificadas por la Federación Nacional de Asociaciones de Personas, y Amigos de Personas con Discapacidad de la República de Panamá, en el mensaje se le pedía que: (a) fomentarán la participación en el estudio de todas las organizaciones que forman parte de Inclusión Panamá; y/o (b) que les ayudaran en el desarrollo de la encuesta; (c) que la encuesta sería por Ilamada telefónica. Se recogió la posibilidad de que un familiar o profesional ayudará a contestar la encuesta en caso de que la persona DID precisara ayuda para ellos en calidad de "apoyo natural". Se solicitó también a las organizaciones que difundieran el estudio ente las familias y profesionales. 
Con el objetivo de que participaran las personas con DID y conocer sus opiniones de las propias personas, el uso y utilidad de las tecnologías y detectar estrategias más efectivas, con objeto de incrementar nuestra contribución a los importantes retos para el empleo de las personas con discapacidad. Diseñar un mapeo nacional sobre competencias en herramientas tecnológicas en las personas con discapacidad hacia una inserción laboral, todos vinculados a asociaciones (Inclusión Panamá, Inclusión Herrera, Inclusión Colón, Inclusión Veraguas, Inclusión Bocas, Inclusión Chiriquí, Down Panamá, Fundación Síndrome Down Ojotos de Amor, Olimpiadas Especiales, Fundación conóceme, Fundación enséñame a vivir, Fundación Laboral JADIS, Fundación para el Desarrollo de la Integración Comunitaria).

La encuesta, creada a partir de la aplicación Google Forms, se alojó en el servidor del dominio Investiga y Educa punto com. El procedimiento de envío y contacto con las personas con discapacidad DID, fue uno a uno por medio de llamadas de teléfono desde la aplicación WhatsApp. El consentimiento informado se incluyó en la encuesta y se acompañó de información sobre los objetivos del estudio, incluyendo un correo electrónico de contacto en el que formular preguntas y obtener información adicional sobre el mismo.

\section{RESULTADOS ALCANZADOS}

Descripción de variables, se han recogido 6 bloques: (1) Aspecto laboral y acceso a tecnología; (2) Tecnologías de uso diario; (3) Usabilidad; (4) Retos de tecnología; (5) Obstáculos de la tecnología; y (6) Herramientas digitales. A continuación, se presentan los resultados de la exploración y descriptiva de estas variables.

El tipo de respuesta varía según la pregunta o bloques: hay respuestas dicotómicas de "si / no"; de opciones (simples o múltiples); y respuestas de escala Likert de 5 puntos.

Aspecto laboral y acceso a tecnología.

En este sub-bloque, nos encontramos con que $57.5 \%$ de los participantes en la encuesta tiene experiencia laboral, aunque solo el $24.4 \%$ se encuentra trabajando en la actualidad. Además, se les ha preguntado que si creen que las tecnologías pueden ayudar a mejorar el empleo y más del 90\% han respondido que sí les ayudarían.

En cuanto a la situación laboral, vemos que hay casi el triple de contratos a tiempo completo que a tiempo parcial (19.2\% vs. $6.8 \%$ ) y que solo el $7.5 \%$ trabaja de forma independiente. Hay un $17.7 \%$ que no dedica su tiempo ni a trabajar ni a estudiar, y un $20.7 \%$ que se encuentra estudiando actualmente. 
Tabla 1: Análisis descriptivo. Aspecto laboral. N=266 participantes

\begin{tabular}{|c|c|c|}
\hline VARIABLE / Categoría & \multicolumn{2}{|c|}{ Frecuencia y $\%$} \\
\hline EXPERIENCIA LABORAL & 153 & $57.5 \%$ \\
\hline TRABAJA ACTUALMENTE & 65 & $24.4 \%$ \\
\hline OTRA ACTIVIDAD LABORAL & 168 & $63.2 \%$ \\
\hline $\begin{array}{l}\text { TECNOLOGIAS PARA MEJORAR EL } \\
\text { EMPLEO }\end{array}$ & 245 & $92.1 \%$ \\
\hline $\begin{array}{l}\text { SITUACIÓN } \\
\text { ACTUAL }\end{array}$ & & \\
\hline Empleado a tiempo completo & 51 & $19.2 \%$ \\
\hline Empleado a tiempo parcial & 18 & $6.8 \%$ \\
\hline Trabajo independiente & 20 & $7.5 \%$ \\
\hline Desempleado & 177 & $66.5 \%$ \\
\hline Busca trabajo & 94 & $35.3 \%$ \\
\hline Ama de casa & 25 & $9.4 \%$ \\
\hline Estudiante & 55 & $20.7 \%$ \\
\hline No trabajo / No estudio & 47 & $17.7 \%$ \\
\hline
\end{tabular}

\section{Acceso a tecnología}

En la siguiente parte del cuestionario se ha preguntado si actualmente tienen acceso a alguna de estas tecnologías: televisión, radio, computadora, tableta, teléfono en casa, teléfono celular, contrato de internet o redes sociales.

Según los resultados mostrados en la tabla 3, podemos ver que las tecnologías que la mayoría de las personas poseen son: Teléfono celular (91.4\%), Televisión (88.7\%) y Radio (67.7), siendo estas las que se corresponden con las más comunes hoy en día. En cuento a las que menos tienen acceso están Internet público (19.2\%), Tableta (20.3\%) y Teléfono en casa (33.85\%). Es importante decir que tan solo el 3.8\% de los participantes no tienen acceso a ninguna de estas tecnologías.

Tabla 2: Análisis descriptivo. Acceso a la tecnología. N=266 participantes

\begin{tabular}{lll}
\multicolumn{2}{c}{ VARIABLE } & \multicolumn{2}{c}{ Frecuencia y } \\
\hline TELEVISIÓN & 236 & $88.7 \%$ \\
RADIO & 180 & $67.7 \%$ \\
COMPUTADORA & 136 & $51.1 \%$ \\
TABLETA & 54 & $20.3 \%$ \\
TELEFONO EN CASA & 90 & $33.8 \%$ \\
TELEFONO CELULAR & 243 & $91.4 \%$ \\
CONTRATO DE INTERNET & 115 & $43.2 \%$ \\
INTERNET PUBLICO & 51 & $19.2 \%$ \\
REDES SOCIALES & 164 & $61.7 \%$ \\
NINGUNA TECNOLOGIA & 10 & $3.8 \%$ \\
\hline
\end{tabular}




\section{Tecnologías de uso diario}

Teniendo en cuenta la competencia digital se ha pedido que seleccionen en qué medida utiliza cada una de las herramientas de uso diario (HUD) en una escala de respuesta tipo Likert de 5 puntos, cuyos resultados aparecen reflejados en la tabla 4.

Según los valores las medias, podemos ver que todas corresponden a puntuaciones medias-altas, por ello se puede concluir que la mayoría de las personas participantes en la encuesta utilizan con bastante frecuencia diaria todas las herramientas citadas. Como excepción, tenemos la variable escuchar la radio, ya que su media es de 2.88 siendo menor que el resto y ello se debe a que la frecuencia de respuesta más alta aparece en la puntuación Likert más baja; esto puede deberse a que la radio, hoy en día, es una herramienta con menor presencia dentro de la sociedad.

Tabla 3: Análisis descriptivo. HUD: Herramientas de uso diarioN=266 participantes

\begin{tabular}{|c|c|c|c|c|c|c|}
\hline \multirow[b]{2}{*}{ ÍTEM } & \multicolumn{5}{|c|}{$\begin{array}{l}\text { \% de } \\
\text { opción }\end{array}$} & \multirow{2}{*}{$\begin{array}{l}\text { Medi } \\
a\end{array}$} \\
\hline & 1 & 2 & 3 & 4 & 5 & \\
\hline teléfono para llamar & $\begin{array}{l}10 . \\
9\end{array}$ & 4.9 & $\begin{array}{l}14 . \\
9\end{array}$ & $\begin{array}{l}15 . \\
0\end{array}$ & $\begin{array}{l}54 . \\
5\end{array}$ & 3.97 \\
\hline mandar mensaje texto & $\begin{array}{l}15 . \\
4\end{array}$ & 6.0 & $\begin{array}{l}10 . \\
9\end{array}$ & $\begin{array}{l}18 . \\
0\end{array}$ & $\begin{array}{l}49 . \\
6\end{array}$ & 3.80 \\
\hline mandar fotos & $\begin{array}{l}26 . \\
7\end{array}$ & $\begin{array}{l}12 . \\
4\end{array}$ & $\begin{array}{l}15 . \\
8\end{array}$ & 8.6 & $\begin{array}{l}36 . \\
5\end{array}$ & 3.16 \\
\hline reenviar mensajes & $\begin{array}{l}22 . \\
9\end{array}$ & $\begin{array}{l}13 . \\
9\end{array}$ & $\begin{array}{l}15 . \\
4\end{array}$ & $\begin{array}{l}15 . \\
0\end{array}$ & $\begin{array}{l}32 . \\
7\end{array}$ & 3.21 \\
\hline conectar a internet & $\begin{array}{l}10 . \\
9\end{array}$ & 5.3 & $\begin{array}{l}15 . \\
8\end{array}$ & $\begin{array}{l}11 . \\
7\end{array}$ & $\begin{array}{l}56 . \\
4\end{array}$ & 3.97 \\
\hline televisor y programas & $\begin{array}{l}20 . \\
3\end{array}$ & 9.0 & $\begin{array}{l}17 . \\
7\end{array}$ & $\begin{array}{l}11 . \\
3\end{array}$ & $\begin{array}{l}41 . \\
7\end{array}$ & 3.45 \\
\hline programas favoritos & $\begin{array}{l}18 . \\
4\end{array}$ & 9.4 & $\begin{array}{l}12 . \\
8\end{array}$ & $\begin{array}{l}15 . \\
4\end{array}$ & $\begin{array}{l}44 . \\
0\end{array}$ & 3.57 \\
\hline escuchar la radio & $\begin{array}{l}35 . \\
3\end{array}$ & $\begin{array}{l}10 . \\
9\end{array}$ & $\begin{array}{l}13 . \\
9\end{array}$ & $\begin{array}{l}10 . \\
2\end{array}$ & $\begin{array}{l}29 . \\
7\end{array}$ & 2.88 \\
\hline escuchar música & $\begin{array}{l}25 . \\
6\end{array}$ & $\begin{array}{l}12 . \\
0\end{array}$ & $\begin{array}{l}14 . \\
7\end{array}$ & $\begin{array}{l}11 . \\
7\end{array}$ & $\begin{array}{l}36 . \\
1\end{array}$ & 3.21 \\
\hline red social & $\begin{array}{l}15 . \\
0\end{array}$ & 9.4 & $\begin{array}{l}16 . \\
9\end{array}$ & $\begin{array}{l}11 . \\
3\end{array}$ & $\begin{array}{l}47 . \\
4\end{array}$ & 3.67 \\
\hline
\end{tabular}

\section{Nociones básicas del uso de computadora}

Para realizar esta parte del estudio, se debe saber primero las personas que sí tienen computadora. Según las respuestas dadas a esta cuestión: dentro de la muestra hay un $50.4 \%$ de personas que tienen computadora $(n=134)$.

Según esto realizamos un filtrado de casos, considerando solamente a los que tienen computadora, que son los que pueden contestar a este bloque de preguntas.

En función del valor medio de las respuestas de estos 134 participantes vemos que la gran mayoría oscilan entre el 3.16 y 3.96 puntos, lo que indica que dan puntuaciones medias-altas. Como excepciones, tenemos: (1) que en el ítem sobre el uso de Microsoft Excel la media alcanza un 2.82, siendo el valor más bajo se puede rela- 
cionar con que el uso de Excel está más enfocado a usos más especíicicos; (2) mientras que, por el contrario, se encuentra el uso de Microsoft Word que tiene la media más alta de respuesta (4.06), y por tanto lo que más usan nuestros participantes. Así mismo, se observa un valor medio bajo en el uso de la computadora como medio de entretenimiento (2.94).

Tabla 4: Análisis descriptivo. Nociones básicas del uso de computadora. N=134 participantes que tienen computadora (50.4\% sobre el $N$ total de participantes)

\begin{tabular}{|c|c|c|c|c|c|c|}
\hline \multirow[b]{2}{*}{ ÍTEM } & \multicolumn{2}{|c|}{$\begin{array}{l}\% \text { de } \\
\text { opción }\end{array}$} & \multicolumn{3}{|c|}{ espuesta de cada } & \multirow{2}{*}{$\begin{array}{l}\text { Medi } \\
a\end{array}$} \\
\hline & 1 & 2 & 3 & 4 & 5 & \\
\hline USO Y ESCRIBO & 7.5 & 9.0 & $\begin{array}{l}14 . \\
9\end{array}$ & $\begin{array}{l}17 . \\
2\end{array}$ & $\begin{array}{l}51 . \\
5\end{array}$ & 3.96 \\
\hline PARA TRABAJAR & $\begin{array}{l}29 . \\
9\end{array}$ & 9.7 & $\begin{array}{l}12 . \\
7\end{array}$ & $\begin{array}{l}10 . \\
4\end{array}$ & $\begin{array}{l}37 . \\
3\end{array}$ & 3.16 \\
\hline PARA ESTUDIAR & $\begin{array}{l}19 . \\
4\end{array}$ & $\begin{array}{l}12 . \\
7\end{array}$ & $\begin{array}{l}16 . \\
4\end{array}$ & $\begin{array}{l}15 . \\
7\end{array}$ & $\begin{array}{l}35 . \\
8\end{array}$ & 3.36 \\
\hline $\begin{array}{l}\text { COMO } \\
\text { ENTRETENIMIENTO }\end{array}$ & $\begin{array}{l}26 . \\
9\end{array}$ & $\begin{array}{l}14 . \\
9\end{array}$ & $\begin{array}{l}19 . \\
4\end{array}$ & $\begin{array}{l}14 . \\
9\end{array}$ & $\begin{array}{l}23 . \\
9\end{array}$ & 2.94 \\
\hline BUSCAR INFORMACIÓN & 7.5 & 8.2 & $\begin{array}{l}14 . \\
9\end{array}$ & $\begin{array}{l}18 . \\
7\end{array}$ & $\begin{array}{l}50 . \\
7\end{array}$ & 3.97 \\
\hline MICROSOFT WORD & $\begin{array}{l}11 . \\
2\end{array}$ & 1.5 & $\begin{array}{l}14 . \\
2\end{array}$ & $\begin{array}{l}16 . \\
4\end{array}$ & $\begin{array}{l}56 . \\
7\end{array}$ & 4.06 \\
\hline $\begin{array}{l}\text { MICROSOFT POWER } \\
\text { POINT }\end{array}$ & $\begin{array}{l}14 . \\
2\end{array}$ & $\begin{array}{l}10 . \\
4\end{array}$ & $\begin{array}{l}23 . \\
1\end{array}$ & $\begin{array}{l}17 . \\
9\end{array}$ & $\begin{array}{l}34 . \\
3\end{array}$ & 3.48 \\
\hline MICROSOFT EXCEL & $\begin{array}{l}33 . \\
6\end{array}$ & $\begin{array}{l}11 . \\
2\end{array}$ & $\begin{array}{l}18 . \\
7\end{array}$ & $\begin{array}{l}12 . \\
7\end{array}$ & $\begin{array}{l}23 . \\
9\end{array}$ & 2.82 \\
\hline CHARLAS DIGITALES & $\begin{array}{l}20 . \\
1\end{array}$ & 9.7 & $\begin{array}{l}13 . \\
4\end{array}$ & $\begin{array}{l}17 . \\
9\end{array}$ & $\begin{array}{l}38 . \\
8\end{array}$ & 3.46 \\
\hline MEMORIA USB & 9.7 & 9.0 & $\begin{array}{l}11 . \\
9\end{array}$ & $\begin{array}{l}24 . \\
6\end{array}$ & $\begin{array}{l}44 . \\
8\end{array}$ & 3.66 \\
\hline
\end{tabular}

\section{Razones para el no uso.}

Con este bloque de preguntas se pretende averigüar cuáles son los motivos para no usar las herramientas digitales. Se recupera para el análisis a la muestra total de participantes.

Se puede apreciar que todas las variables cuentan con un valor promedio en el rango bajo-medio (tabla 5). Las puntuaciones más elevadas se encuentran en: falta de equipamiento informático (2.63) y falta de destrezas (2.53) que son, por tanto, los dos motivos de mayor peso en este no uso de tecnologías. 
Tabla 5: Análisis descriptivo. Razones del no uso. N=266 participantes

\begin{tabular}{|c|c|c|c|c|c|c|}
\hline \multirow[b]{2}{*}{ ÍTEM } & \multicolumn{5}{|c|}{$\begin{array}{l}\% \text { de } \\
\text { opción }\end{array}$} & \multirow{2}{*}{$\begin{array}{l}\text { Medi } \\
a\end{array}$} \\
\hline & 1 & 2 & 3 & 4 & 5 & \\
\hline FALTA DE DESTREZAS & $\begin{array}{l}37 . \\
2\end{array}$ & $\begin{array}{l}13 . \\
9\end{array}$ & $\begin{array}{l}22 . \\
9\end{array}$ & $\begin{array}{l}10 . \\
2\end{array}$ & $\begin{array}{l}15 . \\
8\end{array}$ & 2.53 \\
\hline $\begin{array}{l}\text { NO DISPONIBILIDAD DE } \\
\text { CONEXIÓN }\end{array}$ & $\begin{array}{l}43 . \\
2\end{array}$ & $\begin{array}{l}10 . \\
9\end{array}$ & $\begin{array}{l}18 . \\
8\end{array}$ & 7.5 & $\begin{array}{l}19 . \\
5\end{array}$ & 2.49 \\
\hline $\begin{array}{l}\text { NO EQUIPAMIENTO } \\
\text { INFORMATICO }\end{array}$ & $\begin{array}{l}45 . \\
9\end{array}$ & 8.6 & 9.4 & 8.6 & $\begin{array}{l}27 . \\
4\end{array}$ & 2.63 \\
\hline FALTA DE MOTIVACION & $\begin{array}{l}48 . \\
9\end{array}$ & $\begin{array}{l}13 . \\
9\end{array}$ & $\begin{array}{l}20 . \\
7\end{array}$ & $\begin{array}{l}10 . \\
5\end{array}$ & 6.0 & 2.11 \\
\hline NO NECESARIO & $\begin{array}{l}54 . \\
9\end{array}$ & $\begin{array}{l}12 . \\
0\end{array}$ & $\begin{array}{l}19 . \\
9\end{array}$ & 7.5 & 5.6 & 1.97 \\
\hline
\end{tabular}

\section{Usabilidad.}

En cuanto al uso de internet de los encuestados se les ha preguntado sobre su: (1) uso habitual de internet, (2) la frecuencia de acceso y (3) las formas de conectarse.

1. Uso habitual de internet. El general sus medias oscilan entre valores medios (2.75 y 3.91). Cabe destacar que la concentración más alta de puntos se encuentra en el valor 5 , siendo este el valor más alto de la escala Likert.

2. Frecuencia de acceso. Casi el $50 \%$ de los encuestados han respondido con el valor más alto de esta escala (5), lo que significa que la gran mayoría accede a internet con bastante frecuencia.

3. Formas de conectarse. En este bloque se encuentra una diferencia importante ya que la mitad de las personas del estudio acceden a internet mediante zona wifi de manera habitual. 
Tabla 6: Análisis descriptivo. Uso de internet. $N=266$ participantes

\begin{tabular}{|c|c|c|c|c|c|c|}
\hline \multirow[b]{2}{*}{ ÍTEM } & \multicolumn{5}{|c|}{$\begin{array}{l}\text { \% de respuesta de cada } \\
\text { opción }\end{array}$} & \multirow{2}{*}{$\begin{array}{l}\text { Medi } \\
a\end{array}$} \\
\hline & 1 & 2 & 3 & 4 & 5 & \\
\hline $\begin{array}{l}\text { USO } \quad \text { DE } \\
\text { tengo formación }\end{array}$ & $\begin{array}{l}10 . \\
9\end{array}$ & $\begin{array}{l}13 . \\
5\end{array}$ & $\begin{array}{l}21 . \\
1\end{array}$ & $\begin{array}{l}19 . \\
9\end{array}$ & $\begin{array}{l}34 . \\
6\end{array}$ & 3.54 \\
\hline aprender experimentando & 6.0 & 9.8 & $\begin{array}{l}16 . \\
2\end{array}$ & $\begin{array}{l}23 . \\
7\end{array}$ & $\begin{array}{l}44 . \\
4\end{array}$ & 3.91 \\
\hline cursos sobre tecnología & $\begin{array}{l}34 . \\
6 \\
\end{array}$ & $\begin{array}{l}15 . \\
4\end{array}$ & $\begin{array}{l}13 . \\
9\end{array}$ & $\begin{array}{l}12 . \\
4\end{array}$ & $\begin{array}{l}23 . \\
7\end{array}$ & 2.75 \\
\hline $\begin{array}{l}\text { FRECUENCIA DE ACCESO } \\
\text { varias veces al dia }\end{array}$ & $\begin{array}{l}11 . \\
3\end{array}$ & 7.9 & $\begin{array}{l}16 . \\
2\end{array}$ & $\begin{array}{l}21 . \\
1\end{array}$ & $\begin{array}{l}43 . \\
6\end{array}$ & 3.78 \\
\hline Todos los días & 9.8 & 8.3 & $\begin{array}{l}18 . \\
0\end{array}$ & $\begin{array}{l}18 . \\
8\end{array}$ & $\begin{array}{l}45 . \\
1\end{array}$ & 3.81 \\
\hline Varias veces a la semana & $\begin{array}{l}12 . \\
4\end{array}$ & 6.4 & $\begin{array}{l}15 . \\
8\end{array}$ & $\begin{array}{l}19 . \\
9\end{array}$ & $\begin{array}{l}45 . \\
5\end{array}$ & 3.80 \\
\hline $\begin{array}{l}\text { FORMAS DE CONECTARSE } \\
\text { Zonas wifi }\end{array}$ & $\begin{array}{l}26 . \\
7\end{array}$ & 3.0 & 6.8 & $\begin{array}{l}12 . \\
8\end{array}$ & $\begin{array}{l}50 . \\
8\end{array}$ & 3.58 \\
\hline Prepago & $\begin{array}{l}38 . \\
0\end{array}$ & $\begin{array}{l}17 . \\
3\end{array}$ & $\begin{array}{l}15 . \\
8\end{array}$ & 7.1 & $\begin{array}{l}21 \\
8\end{array}$ & 2.58 \\
\hline Contrato residencial & $\begin{array}{l}46 . \\
2\end{array}$ & 3.0 & 4.9 & 7.9 & $\begin{array}{l}38 . \\
0\end{array}$ & 2.88 \\
\hline
\end{tabular}

\section{Uso y utilidad de herramientas digitales (UUH).}

En este bloque hemos encontrado la media más alta en la pregunta de si usan mensajería instantánea (3.76) puesto que la mayoría de las puntuaciones se encuentran entre los valores altos (4 y 5) y que el $42.1 \%$ de los encuestados respondió con el valor más alto de la escala Likert. El valor medio más bajo (1.77) pertenece a si compran online donde el 71.4\% respondió con Likert más baja (1); a éste le sigue el uso para leer noticias (2.84). El resto, se encuentran entre los valores promedio: 3.30 y 3.70 puntos.

Tenencia de dispositivos.

A los participantes del estudio, se les preguntas sobre si tienen o no tienen los dispositivos que aparecen en el listado de la tabla 9. El dispositivo que más abunda entre estas personas es el celular propio (casi un 90\%), mientras que por el contrario solo un 3\% dispone de un iPod. Se puede destacar respecto a los resultados obtenidos que hay más personas que tienen un laptop (35.0\%) en vez de una computadora fija (26.3\%).

\section{Retos de la tecnología.}

Búsqueda de información a través de internet.

Los ítems de este grupo de nuevo son de tipo Likert. Según las medias de los resultados obtenidos (tabla 10) la mayoría de las personas que participan en la encuesta utiliza el internet como fuente principal para buscar información ya que su valor medio es el más elevado (3.64) que se debe a que se concentra la mayor parte de las respuestas en el valor más alto de la escala Likert (39.1\%).

Acceso a la tecnología a personas con discapacidad intelectual.

A la vista de los valores medios obtenidos, se comprueba que el valor más alto se encuentra en el acceso a internet por recursos propios (3.68) con casi un 50\% que ha respondido con el máximo valor Likert, lo que nos lleva a concluir que la mayoría de las personas disponen de acceso por este medio. Se puede destacar que la 
siguiente media más alta es el no contar con tecnología (2.05) con un elevado número de casos (56.0\%) que ha emitido la respuesta más baja de la escala; en tanto que el resto de ítems tienen valores promedio inferiores a los 2 puntos.

\section{DISCUSIÓN}

El desarrollo de la tecnología ha hecho posible la aparición del teletrabajo, realizando las tareas requeridas por el lugar de trabajo remoto mediante el uso de las TIC (Balbinder y Maciel, 2009). Dada la situación provocada por el COVID-19, el teletrabajo se ha consolidado en las organizaciones, ya que presenta numerosas ventajas para los empleados, las organizaciones y la sociedad frente al trabajo presencial, como mayor autonomía para los trabajadores, eliminación del absentismo, reducción de recursos, eliminación del control de tiempos, expansión geográfica de la organización o reducción del número de contagios por menor movimiento de personas (Santillán, 2020).

El teletrabajo tiene características que aumentan las posibilidades de que las personas con discapacidad consigan un empleo, aumentando así su empleabilidad, ya que las personas con este tipo de trabajos realizan sus tareas desde casa, la organización no necesita invertir tanto en la adecuación física de su sitio y elimina las principales barreras que impiden la contratación de personas con discapacidad (Igeltjørn y Habib, 2020; Me hur y Ulusoy, 2013; Murray, y Kenny, 1990). Murray y Kenny (199), destacaron que el teletrabajo crea nuevas oportunidades para las personas con discapacidades severas.

En general, se han obtenido puntuaciones altas para las herramientas dadas (medias comprendidas entre 3.70 y 4.23), lo que significa que la mayoría de las personas si creen que herramientas como Whatsapp, computadora, plataformas digitales y correo electrónico apoyan al trabajo. Como excepción tenemos a las variables Televisión y Google drive que son las dos que menos puntuación han obtenido (2.82 y 3.16).

Según los valores de las medias de estos ítems: en la variable medio para aprender se encuentra el valor más alto (4.38) debido a que las respuestas obtenidas se reparten entre los Likert más altos (4 y 5), por lo que lo consideran un buen medio para aprender.

En cuanto al valor más bajo se corresponden con las variables: (1) las tecnologías complican la vida: 2.71 puntos; (2) es un factor de exclusión: 2.76 puntos y (3) la tecnología se adapta a las personas: 2.83 puntos; debido a que la mayoría de las respuestas se encuentran en torno a los valores Likert 1, 2 y 3 . Esto quiere decir que gran parte de las personas están en desacuerdo con estas cuestiones. Por último, se les ha dado una serie de herramientas para que valorasen en qué medida pueden apoyar al trabajo.

\section{CONCLUSIONES}

Luego de realizada la investigación se retoman los objetivos planificados, el análisis realizado nos muestra la existencia de una relación significativa $(p<.001)$ con un grado de intensidad moderado (.26); pero suficiente como para poder concluir que las variables están relacionadas entre sí. La relación se define por la asociación de los valores altos entre sí, lo que nos lleva a la conclusión de que tienden a estar más satisfechos aquellos que están más en acuerdo con la afirmación de que las tecnologías son un factor de exclusión. No obstante, también se aprecia una cierta presencia de casos cuyas respuestas son asocian al contario: más satisfacción personal estando menos de acuerdo con la afirmación de exclusión.

\section{REFERENCIAS BIBLIOGRÁFICAS}

Alper, M. y Goggin, G. (2017). Tecnología digital y derechos en la vida de los niños con discapacidad. Nuevos medios y sociedad, 19 (5), 726-740. https://doi.org/10.1177/1461444816686323

Anderberg , P. y Jönsson , B. (2005). Estando allí. Discapacidad y sociedad, 20 (7), 719-733.

Balbinder, M. y Maciel, P. (2009). Telecapacitados: Teletrabajo para la inclusión laboral de personas con discapacidad. Anuario Electrónico de Estudios en Comunicación Social « Disertaciones », 2 (1), 208-237. 
Balladares -Burgos, J. (2019). Competencias para una inclusión educativa digital. Revista PUCE , 191-211. Doi: 10.26807 / revpuce.v0i107.179

Barlott, T., Aplin, T., Catchpole, E. , Kranz, R., Le Goullon, D., Toivanen, A. y Hutchens, s. (2019). Conectividad y TIC: abriendo la puerta a posibilidades para las personas con discapacidad intelectual . Revista de discapacidades intelectuales, 24 (4), 503-521. doi: 10.1177 (14744629519831566

Benavides, CAC, Arias, LFG, Méndez, NDD y Carranza, DA0 (2018). IMU-Mouse: Diseño e implementación de un dispositivo dirigido al desarrollo de interfaces adaptativas para personas con discapacidad física. TecnoLógicas, 21 (41), 63-79.

Berrío Zapata, C., Chaves dos Santos, ZE, Chalhub Oliveira, T., Berrío Zapata, C., Chaves dos Santos, ZE y Chalhub Oliveira, T. (2020). Exclusión digital de las comunidades de personas con discapacidad en Brasil. Revista Cubana de Información en Ciencias de la Salud, 31 (4).

Chacón-Penagos, AM, Ordóñez-Córdo-ba, JA y Anichiarico-González, AM (2017). Hacia el reconocimiento de la inclusión digital como un derecho fundamental en Colombia. Vniversitas (134), 139-168.

Igeltjørn A., Habib L. (2020) ¿El teletrabajo a domicilio como herramienta para la inclusión? Una revisión de la literatura sobre el teletrabajo, las discapacidades y el equilibrio entre la vida personal y laboral. En: Antona M., Stephanidis C. (eds) Universal Access in Human-Computer Interaction. Aplicaciones y práctica. HCII 2020 ( págs. 420-436 ). Lecture Notes in Computer Science, vol 12189. Springer, Cham. https://doi.org/10.1007/978-3-030-49108-6_30

Khanlou , N., Khan, A., Vazquez, LM y Zangeneh, M. (2021). Alfabetización digital, acceso a la tecnología e inclusión para adultos jóvenes con discapacidades del desarrollo. Revista de discapacidades físicas y del desarrollo, 33 (1), 1-25. https://doi.org/10.1007/s10882-020-09738-w

Khetarpal , A. (2015). Tecnología de la información y la comunicación (TIC) y discapacidad. Revisión de la integración del mercado, 6 (1), 96-113. Doi: 10.1177 / 0974929214560117

Leão, M. y Silva, L. (2012). Vivências de trabalhadores com deficiência: uma análise à luz da Psicodinâmica do Trabalho. Revista brasileira. Saúde ocupacional, 37, 159-169

Leonardi, M., Bickenbach, J., Ustun, TB, Kostanjsek, N. y Chatterji, S. (2006). La definición de discapacidad: ¿qué hay en un nombre? The Lancet, 368 (9543), 1219-1221.

Lopez, P. y Samek, T. (2009). Inclusión digital: un nuevo derecho humano. Educación y Bi-blioteca (172), 114118 .

Lorah , ER, Parnell, A., Whitby, PS y Hantula , D. (2015). Una revisión sistemática de las tabletas y los reproductores multimedia portátiles como dispositivos generadores de voz para personas con trastorno del espectro autista. Revista de autismo y trastornos del desarrollo, 45 (12), 3792-3804. https://doi.org/10.1007/s10803014-2314-4

Michailakis , D. (2001). Las tecnologías de la información y la comunicación y las oportunidades de las personas discapacitadas en el mercado laboral sueco , 16 (4), 477-500. D0I: 10.1080 / 09687590120059496

Mishra, M. Sharma, V. y Tripathi, R. (2010). Las TIC como herramienta de enseñanza y aprendizaje en relación con el alumno con discapacidad. Commonwealth of Learning - Aprendizaje para el desarrollo sostenible. Obtenido de http://oasis.col.org/handle/11599/2165 Consultado el 10 de marzo de 2021

Murray , B . Y Kenny , S. (1990). El teletrabajo como opción laboral para personas con discapacidad. Revista internacional de investigación sobre rehabilitación, 13 (3): 205-214. D0I: 10.1097 / 00004356-19900900000003.

Román-Graván, P., Bersabé-Granada, L. y Siles-Rojas, C. (2020). La formación docente universitária em TIC sobre diversidade funcional cognitiva. En Trujillo Torres, JM, Alonso García, S., Campos Soto, MN \& Sola Reche, JM, Análisis sobre metodologias activas y TIC para la enseñanza y el aprendizaje (pp. 524532). Madrid: Dykinson. ISBN: 9788413771731

Santos, BA (2006). Ciberleitura, o contributo das TIC para a leitura no 1CEB . Oporto: Profedições. 
Siles-Rojas, C., Perea-Rodríguez, E. y Román-Graván, P. (2020). Diseño de uma intervención para la inclusión de alumnado con transtorno del espectro autista mediante la robótica educativa. En Trujillo Torres, JM, Alonso García, S., Campos Soto, MN \& Sola Reche, JM, Análisis sobre metodologías activas y TIC para la enseñanza y el aprendizaje (págs. 533-543). Madrid: Dykinson. ISBN: 9788413771731

Sunkel, G. (2012). Buenas prácticas de TIC para una educación inclusiva en América Latina. En G. Sunkel \& D. Trucco, Las tecnologías digitales frente a los desafíos de una educación inclusiva en América Latina: Algunos casos de buenas prácticas (págs. 27-50). Santiago de Chile: CEPAL, Naciones Unidas.

Tanis, S., Palmer, S., Wehmeyer , M., Davies, K., Stock, E., Lobb, K. y Bishop, B. (2012). Encuesta de autoinforme basada en computadora sobre el uso de tecnología por personas con discapacidades intelectuales y del desarrollo. Discapacidades intelectuales y del desarrollo, 50, 53-68. doi: 10.1352 / 1934-9556-50.1.53

UNESCO. (2009). La UNESCO y Sun Microsystems anuncian un esfuerzo conjunto de educación y desarrollo comunitario impulsado por tecnologías abiertas. Comunicado de prensa de la UNESCO. Disponible en http://www.lacult.unesco.org/noticias/showitem.php?uid_(consultado el 10 de enero de 2021)

Naciones Unidas (2017). Comité de Derechos de las Personas con Discapacidad considera informe inicial de Panamá. Disponible en https://www.ohchr.org/en/NewsEvents/Pages/DisplayNews.a

Comité Warnock. (1978). Necesidades educativas especiales: Informe Warnock. Londres: DES

Zúniga, S., Sandoval, J., Martínez, V. \& Cuervo, A. (2016). Percepción de estudiantes con discapacidad visual sobre sus competencias digitales en una universidad pública del sureste de México. Apertura, Revista de Innovación Educativa, 8 (1). 